\title{
Enhancement of Bioavailability and Pharmacodynamic Effects of Thymoquinone Via Nanostructured Lipid Carrier (NLC) Formulation
}

\author{
Mohammed Elmowafy, ${ }^{1,6}$ Ahmed Samy, ${ }^{1}$ Mohamed A. Raslan, ${ }^{1}$ Ayman Salama, ${ }^{1}$ Ragab A. Said, ${ }^{2}$ \\ Abdelaziz E. Abdelaziz, ${ }^{3}$ Wafaa El-Eraky, ${ }^{4}$ Sally El Awdan, ${ }^{4}$ and Tapani Viitala ${ }^{5}$
}

Received 12 May 2015; accepted 9 August 2015; published online 25 August 2015

\begin{abstract}
Thymoquinone (TQ), obtained from black cumin (Nigella sativa), is a natural product with antioxidant, anti-inflammatory, and hepatoprotective effects but unfortunately with poor bioavailability. Aiming to improve its poor oral bioavailability, TQ-loaded nanostructured lipid carriers (NLCs) were prepared by high-speed homogenization followed by ultrasonication and evaluated in vitro. Bioavailability and pharmacodynamic studies were also performed. The resultant NLCs showed poor physical homogeneity in Compritol 888 ATO Pluronic F127 system which consequently produced larger particle size and polydispersity index, smaller zeta potential values, and lower short-term (30 days) physical stability than other systems. Encapsulation efficiency percentage (EE\%) lied between $84.6 \pm 5 \%$ and $96.2 \pm 1.6 \%$. TQ $\mathrm{AUC}_{0-t}$ values were higher in animals treated with NLCs, with a relative bioavailability of 2.03- and 3.97fold (for F9 and F12, respectively) higher than TQ suspension, indicating bioavailability enhancement by NLC formulation. Hepatoprotective effects of F12 showed significant $(P<0.05)$ decrease in both serum alanine amino transferase and aspartate amino transferase to reach $305.0 \pm 24.88$ and $304.7 \pm 23.55 \mathrm{U} / \mathrm{ml}$, respectively, when compared with untreated toxic group. Anti-oxidant efficacy of F12 showed significant $(P<0.05)$ decline of malondialdehyde and elevation of reduced glutatione. This improvement was also confirmed histopathologically.
\end{abstract}

KEY WORDS: bioavailability; hepatoprotective activity; NLC; thymoquinone.

\section{INTRODUCTION}

Thymoquinone (TQ), chemically named 2-isopropyl-5methyl-1,4-benzoquinone (Fig. 1), is the main active constituent of Nigella sativa plant oil, also known as black seed or black cumin (1). The N. sativa essential oil has been traditionally used in folk medicine due to its essential therapeutic effects. Recently, several studies have shown that TQ has many pharmacological effects, including anti-oxidation and hepatoprotective effects against hepatotoxins $(2,3)$, neuroprotective (4), anti-diabetic (5), anti-inflammatory (6), antimutagenic (7), and anti-carcinogenic (8). A recent study by Oguz et al. shows that TQ inhibits common bile duct ligation (CBDL)-induced liver damage in rats including the fibrotic changes in the liver (9). So far, the hepatoprotective and anti-

\footnotetext{
${ }^{1}$ Department of Pharmaceutics and Industrial Pharmacy, Faculty of Pharmacy (Boys), Al-Azhar University, Nasr City, Cairo, Egypt.

${ }^{2}$ Department of Analytical Chemistry, Faculty of Pharmacy (Boys), Al-Azhar University, Nasr City, Cairo, Egypt.

${ }^{3}$ Department of Pharmaceutics, Faculty of Pharmacy and Pharmaceutical Manufacturing, Kafr Elsheikh University, Kafr Elsheikh, Egypt.

${ }^{4}$ Pharmacology Department, National Research Center, Giza, Egypt.

${ }^{5}$ Division of Pharmaceutical Biosciences, Faculty of Pharmacy, University of Helsinki, Helsinki, Finland.

${ }^{6}$ To whom correspondence should be addressed. (e-mail: morere_om@yahoo.com)
}

fibrotic effects of TQ is largely attributed to its anti-oxidant activity, which leads to decreased hepatocyte damage and thus decreased transactivation of hepatic stellate cells (HSCs). However, the detailed mechanism remains incompletely understood. Particularly, a role of TQ in directly inhibiting the fibrogenic activity of HSCs has not been studied. Although being an excellent drug for treating liver diseases, its use is limited owing to its poor aqueous solubility and bioavailability.

Lipid-based drug delivery systems have the capability to improve the solubility and bioavailability of oral administered poorly water-soluble and/or lipophilic drugs (10). The first generation of lipid nanoparticles, called solid lipid nanoparticles (SLN), is formulated by dispersing the nanoparticles of solid lipid matrix into an aqueous dispersion stabilized by one or more emulsifying agent. Drawbacks of SLN, as lowered drug-loading capacity and drug leakage from lipid core during storage due to recrystallization of solid lipid, enforce into creating the second generation of lipid nanoparticles, the nanostructured lipid carriers (NLC). As NLC dispersions are formed by a mixture of both solid lipid and liquid lipid, they exhibit a higher drug payload and little drug expulsion during storage $(1,11)$. This higher drug encapsulation is attributed to the differences in the structures of the solid and liquid lipids, and then formation of a perfect crystal is distorted. Thus, the mixture accommodates the active in molecular form or in amorphous clusters (12). Additionally, NLCs promote oral 


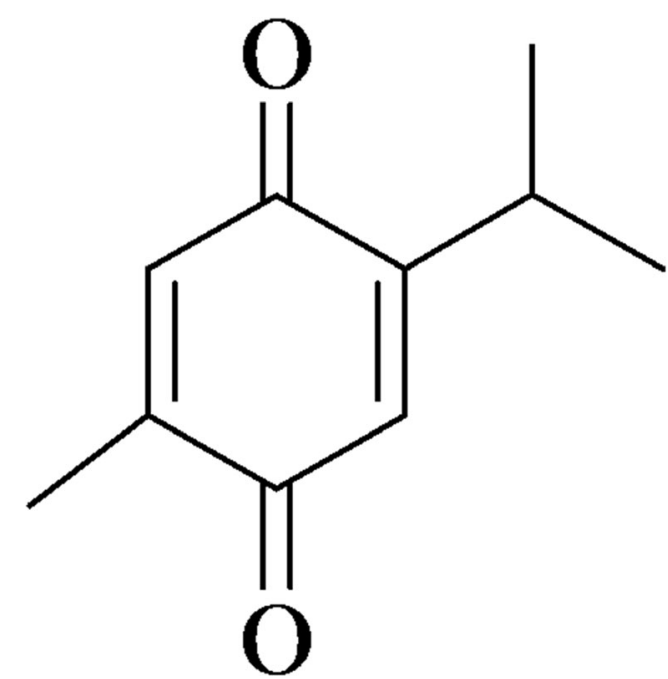

Fig. 1. Chemical structure of TQ

absorption of encapsulated drug via selective uptake through lymphatic route or payer's patches $(13,14)$.

The objective of the present work was to load TQ into NLCs in order to enhance oral absorption and hence bioavailability. Compritol 888 ATO and Gelucire 43/01 were chosen as solid lipids while Miglyol was liquid lipid. Two different hydrophilic surfactants (Tween 80 and Pluronic F27) were investigated while lecithin was the lipophilic emulsifier. The TQloaded NLCs were developed and screened according to the physicochemical characteristics. Based on the outcomes of preformulation studies, the selected TQ-loaded NLCs were subjected to in vivo evaluation to elucidate their feasibility in enhancing the bioavailability and hepatocurative efficacy.

\section{MATERIALS AND METHODS}

\section{Materials}

TQ and Pluronic F127 were purchased from SigmaAldrich (Germany). Miglyol 812 (capriylic/capric triglycerides) was purchased from Caelo, Hilden (Germany). Compritol 888 ATO, Gelucire43/01, Suppocire A and Geleol were kindly gifted by Gattefosse (France). Lecithin (S75) was purchased from Lipoid (Germany). Cacao butter and Witepsol E75 were purchased from Morgan Chemicals Co. (Egypt). Tween 80 was purchased from ADWIC Chemicals Co. (Egypt). All the other chemicals, reagents, and solvents used were of analytical reagent grade.

\section{Methods}

\section{Compatibility Between Lipid Components}

A compatibility screening of liquid lipid with solid lipids in ratios 1:4, 1:1, and 4:1 were performed. The lipid mixtures used were namely Compritol 888 ATO/Miglyol, Gelucire 43/ 01/Miglyol, Suppocire A/Miglyol, Geleol/Miglyol, cacao butter/Miglyol, and Witepsol E75/Miglyol. Both solid and liquid lipids were accurately weighed into glass vials and heated up to $100^{\circ} \mathrm{C}$. The melts were checked after $1 \mathrm{~h}$, immediately after solidification and after $24 \mathrm{~h}$. Mixtures of one single phase only were selected for preparation step.

\section{Preparation of TQ-Loaded NLCs}

The NLC was prepared by a modified method of highspeed homogenization followed by ultrasonication. The lipid and aqueous phases were prepared separately. The solid lipid/ liquid lipid phase consisted of $10 \%(w / v)$ Compritol (or Gelucire 43/01) and Miglyol at different ratios and $0.5 \%$ lecithin as the lipophilic stabilizer, while the aqueous phase consisted of distilled water and $2 \%$ hydrophilic emulsifier (Tween 80 or Pluronic F127). TQ was firstly dissolved in the lipid phase. Both phases were heated separately to $85^{\circ} \mathrm{C}$ for $10 \mathrm{~min}$. The aqueous phase was added dropwise to the molten lipid phase and mixed using a high-speed homogenizer (Jahnke \& Kunkel, GmbH, Staufen, Germany) at $10,000 \mathrm{rpm}$ for $10 \mathrm{~min}$. The mixture was further treated using a probe-type sonicator (4710 series-Crest Ultrasonic Corp, New York, USA) for $10 \mathrm{~min}$ at $50 \mathrm{~W}$. The obtained dispersion cooled at room temperature. The formulations are depicted in Table I.

\section{Drug Encapsulation Efficiency}

The encapsulation efficiency (EE) is referring to the ratio of the concentration of TQ entrapped in the lipid core to that of the whole amount of TQ added during NLC preparation. The proportion of non-encapsulated TQ was determined by centrifugation precipitation method. NLC aggregation was obtained by adjusting the $\mathrm{pH}$ value of the NLC dispersion to 1.20 which could be achieved by adding $0.1 \mathrm{~N}$ of hydrochloric acid. Then the precipitate was precisely separated by centrifugation at $21,000 \times g$ for $15 \mathrm{~min}$ (Biofuge primo centrifuge, England) followed by filtration through $0.45 \mu \mathrm{m}$ filter. The filtrate was diluted appropriately with ethanol and analyzed by UV-visible spectrophotometer (Shimadzu, Japan) at $254 \mathrm{~nm}$. Calibration curve for the validated UV assay of TQ was constructed using five TQ concentrations ranges of 0.5$5 \mu \mathrm{g} / \mathrm{ml}$. Correlation coefficient was $>0.999$. Each point was repeated twice and the average was used in construction whereas the error was calculated as standard deviation $( \pm$ SD). The encapsulation efficiency of TQ was then calculated according to the following Eq. (1):

$\% \mathrm{EE}=(\mathrm{TD}-\mathrm{UD} / \mathrm{TD}) \times 100$,

where $\% \mathrm{EE}$ is the encapsulation efficiency in percentage, TD is the total amount of added drug during NLC preparation, and UD is the amount of unencapsulated free drug in the supernatant after centrifugation.

\section{Particle Size and Zeta Potential}

The mean particle size, polydispersity index (PDI), and zeta potential of the TQ-loaded NLC formulations were determined by dynamic light scattering (Zetasizer Nano ZS, Malvern Instruments, UK). All the measurements were made after dilution by 200 -fold with Milli-Q water $(18.2 \mathrm{M} \Omega \mathrm{cm}$, 
Table I. Compositions and Encapsulation Efficiency Percentages (EE\%) of Different Formulations $(n=3, \pm \mathrm{SD})$

\begin{tabular}{|c|c|c|c|c|c|}
\hline Code & Solid lipid & Solid lipid/Miglyol (10\%) & Aqueous surfactant $(2 \%)$ & Lecithin & $\mathrm{EE} \%$ \\
\hline NLC 1 & Compritol ATO 888 & $8 / 2$ & Pluronic F127 & $0.5 \%$ & $84.6 \pm 5$ \\
\hline NLC 2 & Compritol ATO 888 & $5 / 5$ & Pluronic F127 & $0.5 \%$ & $88.5 \pm 2.6$ \\
\hline NLC 3 & Compritol ATO 888 & $2 / 8$ & Pluronic F127 & $0.5 \%$ & $87.6 \pm 9.4$ \\
\hline NLC 4 & Compritol ATO 888 & $8 / 2$ & Tween 80 & $0.5 \%$ & $91.5 \pm 4.3$ \\
\hline NLC 5 & Compritol ATO 888 & $5 / 5$ & Tween 80 & $0.5 \%$ & $93.6 \pm 3.4$ \\
\hline NLC 6 & Compritol ATO 888 & $2 / 8$ & Tween 80 & $0.5 \%$ & $92.7 \pm 2.9$ \\
\hline NLC 7 & Gelucire 43/01 & $8 / 2$ & Pluronic F127 & $0.5 \%$ & $85.3 \pm 6.1$ \\
\hline NLC 8 & Gelucire 43/01 & $5 / 5$ & Pluronic F127 & $0.5 \%$ & $88.5 \pm 8.2$ \\
\hline NLC 9 & Gelucire 43/01 & $2 / 8$ & Pluronic F127 & $0.5 \%$ & $95.6 \pm 2.8$ \\
\hline NLC 10 & Gelucire 43/01 & $8 / 2$ & Tween 80 & $0.5 \%$ & $94.7 \pm 2.9$ \\
\hline NLC 11 & Gelucire 43/01 & $5 / 5$ & Tween 80 & $0.5 \%$ & $93.2 \pm 4.7$ \\
\hline NLC 12 & Gelucire 43/01 & $2 / 8$ & Tween 80 & $0.5 \%$ & $96.2 \pm 1.6$ \\
\hline
\end{tabular}

Millipore, Bedford, USA) at room temperature and measured at a light scattering angle of $90^{\circ}$.

\section{Morphology of NLCs}

The morphology of TQ-loaded NLCs was observed by transmission electron microscopy (TEM; JEOL JEM-1010S Tokyo, Japan). Formulations were diluted with distilled water and placed on a carbon-coated copper grid to form a thin liquid film. Excess of sample was then removed by a filter paper. The films on the grid were allowed to dry at room temperature, and then observed by TEM and photographed.

\section{Short-Term Stability}

In order to check the stability of the prepared systems, particle size, PDI, and zeta potential were measured after 30 days (stored at $4^{\circ} \mathrm{C}$ ). The formulations were also observed visually for a characteristic nanometric dimensions and the absence of lipid particulates immediately after preparations and after 30 days. Partitioning was not expected in NLC formulations during this short period as TQ is completely soluble in Miglyol.

\section{In Vivo Evaluation}

This study was carried out in order to investigate the bioavailability and hepatocurative efficacy of TQ-loaded NLCs when compared with TQ solution after oral administration in rats.

\section{Animals}

Adult male albino rats, weighing 180-250 g, were used in all experiments of this study. They were obtained from the Animal House Colony of the National Research Center (Dokki, Giza, Egypt) and were housed under conventional laboratory conditions throughout the period of experimentation. The study was conducted in agreement with ethics and policies approved by the Animal Care and Use Committee of National Research Center. The animals were fed a standard rat pellet diet and allowed free access to water.

\section{Pharmacokinetic Study}

To perform pharmacokinetic studies, the experiment was carried out with 15 rats $(n=5)$ divided randomly into three groups with five rats each. The first group was orally administered a single dose of TQ $(20 \mathrm{mg} / \mathrm{kg})$ suspension (prepared by dispersing TQ in equal quantities of PEG400 and glycerol). The second and third groups were administered a single dose of NLC9 and NLC12, respectively (equivalent to $20 \mathrm{mg} / \mathrm{kg}$ ). One milliliter of blood was taken from the retro-orbital plexus using light ether as anaesthesia at pre-determined time intervals (pre-dose, 0.5, 1, 2, 3, 4, $8,12,24,48 \mathrm{~h}$ ), put into heparinized Eppendorf tube, and directly centrifuged at $3000 \mathrm{rpm}$ for $15 \mathrm{~min}$ to separate plasma. After centrifugation, the plasma was preserved at $-40^{\circ} \mathrm{C}$ until analysis.

\section{Chromatography}

The plasma concentrations of TQ were determined by a HPLC (LCD Analytical System USA). The stationary phase comprised of Athena C18 (120Á, 4.6×250 mm, $5 \mu \mathrm{m}) \mathrm{CNW}$ Technologies. The chromatographic separation was carried out by isocratic elution using mobile phase consisting of a mixture of acetonitrile and $20 \mathrm{mM} \mathrm{KH}_{2} \mathrm{PO}_{4}$ (adjusted to $\mathrm{pH}$ 4.5 using orthophosphoric acid; $(80: 20, v / v)$ and was pumped at a flow rate of $1 \mathrm{ml} / \mathrm{min}$. The injection volume was $20 \mu \mathrm{l}$, and analysis was performed at $254 \mathrm{~nm}$ wavelength with a total run time of $10 \mathrm{~min}$. Data acquisition, data handling, and instrument control were performed by ChromoQuest Software v4.2.34.

\section{Sample Preparation}

TQ was extracted from the plasma using acetonitrile and methanol as extraction solvents. An aliquot of $200 \mu \mathrm{l}$ of plasma-containing thymoquinone was transferred into a capped centrifuge tube, and then $200 \mu \mathrm{l}$ of thymol $(4 \mu \mathrm{g} / \mathrm{ml}$ as internal standard) was added and precipitated with acetonitrile $(300 \mu \mathrm{l})$ and methanol to make a volume of up to $1 \mathrm{ml}$. The mixture was then shaken and centrifuged at $6000 \mathrm{rpm}$ for another $5 \mathrm{~min}$. And from the supernatant, $20 \mu \mathrm{l}$ was injected onto the column for the analysis of TQ. 


\section{Assay Validation}

The assay procedures were validated in terms of linearity, precision, and accuracy, and recovery studies and limits of detection and quantification were carried out using the plasma standards. The precision (intra- and inter-day) of the method was expressed as the percentage relative standard deviation (RSD\%).

\section{Calculation of TQ Pharmacokinetic Parameters}

The main pharmacokinetic parameters were obtained with the help of a pharmacokinetic program KineticaTM v. 4 software. The time of maximum concentration $\left(T_{\max }\right)$ and values of maximum concentration $\left(C_{\max }\right)$ were directly obtained from the plasma concentration-time curve where the area under the concentration-time curve (AUC) was calculated by linear trapezoidal method. The relative bioavailability of NLC formulations was determined using the following equation:

$$
\% \mathrm{Fr}=(\mathrm{AUCt} . \mathrm{Dr} / \mathrm{AUCr} . \mathrm{Dt})
$$

where Fr was the relative bioavailability, AUC was the area under the plasma concentration-time curve, $D$ was the dose administrated, $t$ was the test formulation (oral administration of TQ-loaded NLC), and $r$ was the reference formulation (oral administration of TQ suspension).

\section{Pharmacological Effects}

Male Wistar rats were randomly divided to four groups of five rats each. Group I (control group) received normal saline $\left(1 \mathrm{ml} \mathrm{kg}{ }^{-1}\right.$ day $\left.^{-1}\right)$ orally once daily for 7 days. Rats of the remaining three groups received $800 \mathrm{mg} / \mathrm{kg}$ paracetamol once daily for 7 days to induce hepatic damage. Group II did not receive TQ and acted as untreated toxic group. Groups III and IV received oral NLC9 and NLC12 (dose equivalent to $20 \mathrm{mg}$ TQ/kg weight) daily for 7 days, respectively, along with paracetamol dose.

On day 8 , animals were anaesthetized by ether inhalation, blood samples were collected in centrifuge tube. Serum was separated from the clotted blood samples by centrifugation at $5000 \mathrm{rpm}$ for $5 \mathrm{~min}$, and then biochemically tested for serum alanine amino transferase (ALT), aspartate amino transferase (AST), total bilirubin, and albumin to assess liver functions (15). The activities of these enzymes were colorimetrically determined using a commercial kit (Siemens Healthcare Diagnostics, Egypt).

Liver tissue homogenates were tested for lipid peroxidation (by malondialdehyde (MDA) level which was measured using the thiobarbituric acid reactive substances (TBARS) assay, as described by Mihara and Uchiyama (16) and hepatic reduced glutathione (GSH) level which was determined colorimetrically at $412 \mathrm{~nm}$ by Ellman reagent (17).

\section{Histopathological Examination}

To verify the investigated liver function results, tissue specimens from livers of different rats groups were fixed in $10 \%$ neutral buffered formalin for $24 \mathrm{~h}$. Then, distilled water was used to wash fixed tissues followed by dehydration in serial dilutions of alcohol. Tissues were cleared in xylene and embedded in paraffin bees wax blocks and kept at $56^{\circ} \mathrm{C}$ for another $24 \mathrm{~h}$. Sections from the paraffin blocks of $4 \mu \mathrm{m}$ thickness were cut by sledge microtome, deparaffinized, and stained with hematoxyline and eosin (18). All sections were examined using an electric light microscope (Binuclear XS2N107T, China).

\section{Statistical Analysis}

The results in this work are expressed as a mean \pm standard deviation (SD). The statistical analysis of obtained results was evaluated by one-way ANOVA followed by Tukey-Kramer test using the StatPlus software (AnalystSoft Inc., USA). Difference at $P<0.05$ was considered to be significant.

\section{RESULTS AND DISCUSSION}

\section{Compatibility Test and Formulations}

Among the solid lipids screened, perfect compatibility with Miglyol was found in both Compritol 888 ATO and Gelucire43/01. Table I shows the compositions of the 12 different TQ-loaded NLCs that were prepared for this study. NLCs (1-6) contained Compritol 888 ATO as solid lipid while NLCs (7-12) contained Gelucire43/01. Formulations were prepared in three different ratios between solid lipid and liquid lipid (4:1, 1:1, and 1:4) aided by addition of fixed percentage $(2 \%)$ from one of two hydrophilic surfactants (Pluronic F127 and Tween 80). Lecithin was added to all formulations (as lipophilic surfactant) to all formulations by the same percentage $(0.5 \%)$. This allowed us to concentrate on how the different types and concentrations of solid lipid and types of hydrophilic surfactant would affect the characteristics of the NLCs.

\section{Encapsulation Efficiency}

The entrapment efficiency of TQ within the different prepared nanostructured formulations was found to vary between $84.6 \% \pm 5$ and $96.2 \% \pm 1.6$ (Table I). It is clear that Gelucire43/01 containing NLCs produced similar drug entrapment efficiencies as Compritol 888 ATO $(P=0.298)$. This may be attributed to complete solubility of TQ in Miglyol which in turn leads to massive crystal order disturbance and leaves enough space to entrap drug molecules, thus leading to enhanced drug entrapment efficiency (19). Furthermore, addition of liquid lipid (Miglyol) tends to impel the formation of a small particle population as result of a higher molecular mobility of the matrix (20). Concerning hydrophilic surfactant, Tween 80 displayed a higher entrapment efficiencies with significant difference when compared with Pluronic F127 $(P=0.012)$. This behavior might be attributed to two factors; the first is higher solubilization effect of Pluronic F127 than Tween 80 (21). The second factor is physical and chemical compatibilities (affinity, miscibility) between the drug-polymer complex and the solid lipid phase (22). These results suggested the feasibility of Tween 80 system more than Pluronic F127 system. It is obvious that increasing the liquid 
Table II. Particle Size, PDI, and Zeta Potential $(n=3, \pm$ SD) of TQ-Loaded NLC Formulations

\begin{tabular}{|c|c|c|c|c|c|c|c|c|}
\hline \multirow[t]{2}{*}{ Code } & \multicolumn{4}{|c|}{ Freshly prepared } & \multicolumn{4}{|c|}{ After 30 days } \\
\hline & $\begin{array}{l}\text { Particle size } \\
\quad(\mathrm{nm})\end{array}$ & PDI & $\begin{array}{l}\text { Zeta potential } \\
\quad(\mathrm{mV})\end{array}$ & Vis. Obs. & Particle size & PDI & $\begin{array}{l}\text { Zeta potential } \\
\quad(\mathrm{mV})\end{array}$ & Vis. Obs. \\
\hline NLC 1 & $528 \pm 42$ & $0.74 \pm 0.3$ & $-39.5 \pm 0.36$ & Rel. clear & $826 \pm 78.39$ & $0.94 \pm 0.1$ & $-37.1 \pm 0.45$ & Particulate \\
\hline NLC 2 & $218.1 \pm 14$ & $0.51 \pm 0.03$ & $-38.8 \pm 0.54$ & Rel. clear & $249.4 \pm 25$ & $0.65 \pm 0.05$ & $-35.8 \pm 0.66$ & Rel. clear \\
\hline NLC 3 & $466.1 \pm 1.7$ & $0.32 \pm 0.02$ & $-41.7 \pm 0.65$ & Rel. clear & $698 \pm 44.4$ & $0.52 \pm 0.01$ & $-47.7 \pm 0.75$ & Particulate \\
\hline NLC 4 & $163.9 \pm 3.6$ & $0.34 \pm 0.02$ & $-40.2 \pm 2.6$ & Rel. clear & $174.1 \pm 3.2$ & $0.36 \pm 0.02$ & $-41.2 \pm 1.43$ & Rel. clear \\
\hline NLC 5 & $165.2 \pm 7.6$ & $0.26 \pm 0.03$ & $-37.4 \pm 1.91$ & Clear susp. & $195 \pm 0.68$ & $0.23 \pm 0.01$ & $-36.8 \pm 1.23$ & Clear susp \\
\hline NLC 6 & $159.2 \pm 36.4$ & $0.23 \pm 0.01$ & $-42.5 \pm 0.25$ & Clear susp. & $166.1 \pm 1.5$ & $0.22 \pm 0.02$ & $-39.1 \pm 0.23$ & Clear susp \\
\hline NLC 7 & $197.6 \pm 6.9$ & $0.39 \pm 0.01$ & $-49.6 \pm 0.85$ & Rel. clear & $265.2 \pm 7.6$ & $0.76 \pm 0.03$ & $-48.8 \pm 0.62$ & Particulate \\
\hline NLC 8 & $176.3 \pm 2.75$ & $0.32 \pm 0.01$ & $-55.3 \pm 0.29$ & Rel. clear & $188.9 \pm 1.15$ & $0.22 \pm 0.01$ & $-56.4 \pm 0.87$ & Rel. clear \\
\hline NLC 9 & $163.1 \pm 3.25$ & $0.2 \pm 0.01$ & $-58.6 \pm 0.5$ & Clear susp. & $179.2 \pm 1.5$ & $0.22 \pm 0.006$ & $-57.8 \pm 0.4$ & Clear susp. \\
\hline NLC 10 & $192.1 \pm 2.8$ & $0.22 \pm 0.002$ & $-56.7 \pm 0.3$ & Clear susp. & $206.6 \pm 1.5$ & $0.23 \pm 0.003$ & $-54.8 \pm 0.6$ & Clear susp \\
\hline NLC 11 & $161.4 \pm 3.68$ & $0.21 \pm 0.01$ & $-55.6 \pm 0.24$ & Clear susp. & $176.5 \pm 1.66$ & $0.23 \pm 0.007$ & $-51.1 \pm 0.55$ & Clear susp \\
\hline NLC 12 & $141.9 \pm 5.1$ & $0.14 \pm 0.01$ & $-54.2 \pm 0.3$ & Clear susp. & $144.1 \pm 1.13$ & $0.15 \pm 0.02$ & $-53.7 \pm 0.4$ & Clear susp \\
\hline
\end{tabular}

lipid portion insignificantly enhances $(P>0.05)$ the encapsulation efficiencies as the smallest percentage $(2 \%)$ was capable of solubilizing added TQ.

\section{Particle Size and Zeta Potential}

Table II shows the particle size, PDI, zeta potential, and visual observation of both freshly prepared and 30 days stored formulations. In case of freshly prepared, the particle size was in the colloidal range and below $500 \mathrm{~nm}$ for all the prepared formulae except NLC1in which Compritol 888 ATO and Pluronic F127 were solid lipids and hydrophilic surfactant, respectively. In our case, formulations contain Compritol 888 ATO and Pluronic F127 exhibited the largest particle size. This behavior might be attributed to incompatibility between both components, which was obvious in EE investigation ("Compatibility Between Lipid Components"), as changing one of them produced smaller particles size. Melting point of solid lipid might be another predisposing factor as the mean particle size of lipid nanoparticles increased with increasing melting point of the matrix constituent, indicating an effect of the melt viscosity (23) as melting point of Compritol 888 ATO $\left(69-74^{\circ} \mathrm{C}\right)$ is higher than Gelucire $43 / 01\left(42-46^{\circ} \mathrm{C}\right)$. As HLB values of Compritol 888 ATO (HLB 2) and Gelucire43/01 (HLB 1) were so close, their effect in improving emulsification and probably influence particle size (24) could be abolished. The particle size distribution of the formulations with higher Miglyol percentage (8\%) and that of Gelucire 43/ 01 and Tween 80 showed very good homogeneity as the PDI values laid between 0.15 and 0.3 . Other formulations looked heterogeneous as PDI $>0.3$ which suggested the instability of these formulations. Usually, a small value of PDI (0.2) indicates a homogenous vesicle population, while a larger PDI $(>0.3)$ means a high heterogeneity in particle size (25). The surface charge of the different samples was consistently negative and ranged from $-37.4 \pm 1.91$ to $-58.6 \pm 0.5 \mathrm{mV}$. The results showed that the type of solid lipid was a critical parameter governing zeta potential as Gelucire43/01 containing NLCs showed higher surface charges $(P<0.05)$ when compared with formulations containing Compritol 888 ATO. It was noted that there was no linear correlation between the zeta potential and solid lipid/liquid lipid ratios.

\section{Morphology}

The morphology of TQ-loaded NLCs determined by TEM was shown in Fig. 2. The TEM image showed that the particles had oval and almost rounded shapes and behaved separated from each others. The mean diameter was in the range of 100-200 nm.

\section{Stability of NLCs}

Stability estimation for formulations was done on basis of particle size, zeta potential, PDI variations, and visual observation for a 1-month period (Table II). Results showed that formulations containing Compritol 888 ATO and Pluronic F127 (NLCs1-3) exhibited extensive particle size growth, heterogeneous particle distributions, and unclear dispersion while NLC12 showed the best stability concerning the investigated parameters. NLC9 showed minimal particle size growth, uniform particle size distribution, absence of visible particulate matter, and the highest zeta potential value among all investigated formulations. Thus, NLC9 and NLC12 were chosen for the following studies. It is clear that the change in zeta potential is negligible.

\section{Pharmacokinetic Study}

\section{Assay Validation of TQ in Rat Plasma}

The calibration curves of TQ in solution and plasma were found to be linear over the selected concentration range of $0.5-$ $8 \mu \mathrm{g} / \mathrm{ml}$ (linear equation, $y=0.786 x+0.331$ and correlation coefficient; $\left.r^{2}=0.999\right)$. Three replicates of LQC, MQC, and HQC samples were processed and analyzed over 3 days for accuracy and precision evaluation by spiking blank plasma with 1,4 , and $8 \mu \mathrm{g}$ of TQ.

The intra-day accuracy ranged from $99.42 \%$ to $101.39 \%$, while the intra-day precision ranged from $0.424 \%$ to $1.154 \%$. The inter-day accuracy ranged from $92.2 \%$ to $100.23 \%$, while the inter-day precision ranged from $0.509 \%$ to $0.714 \%$. The low values of RSD\% and percent of recoveries established the precision and accuracy of the proposed method. The limits of detection and quantification (LOD and LOQ) for plasma samples were found to be 0.115 and $0.383 \mu \mathrm{g} \mathrm{ml}^{-1}$ respectively. 


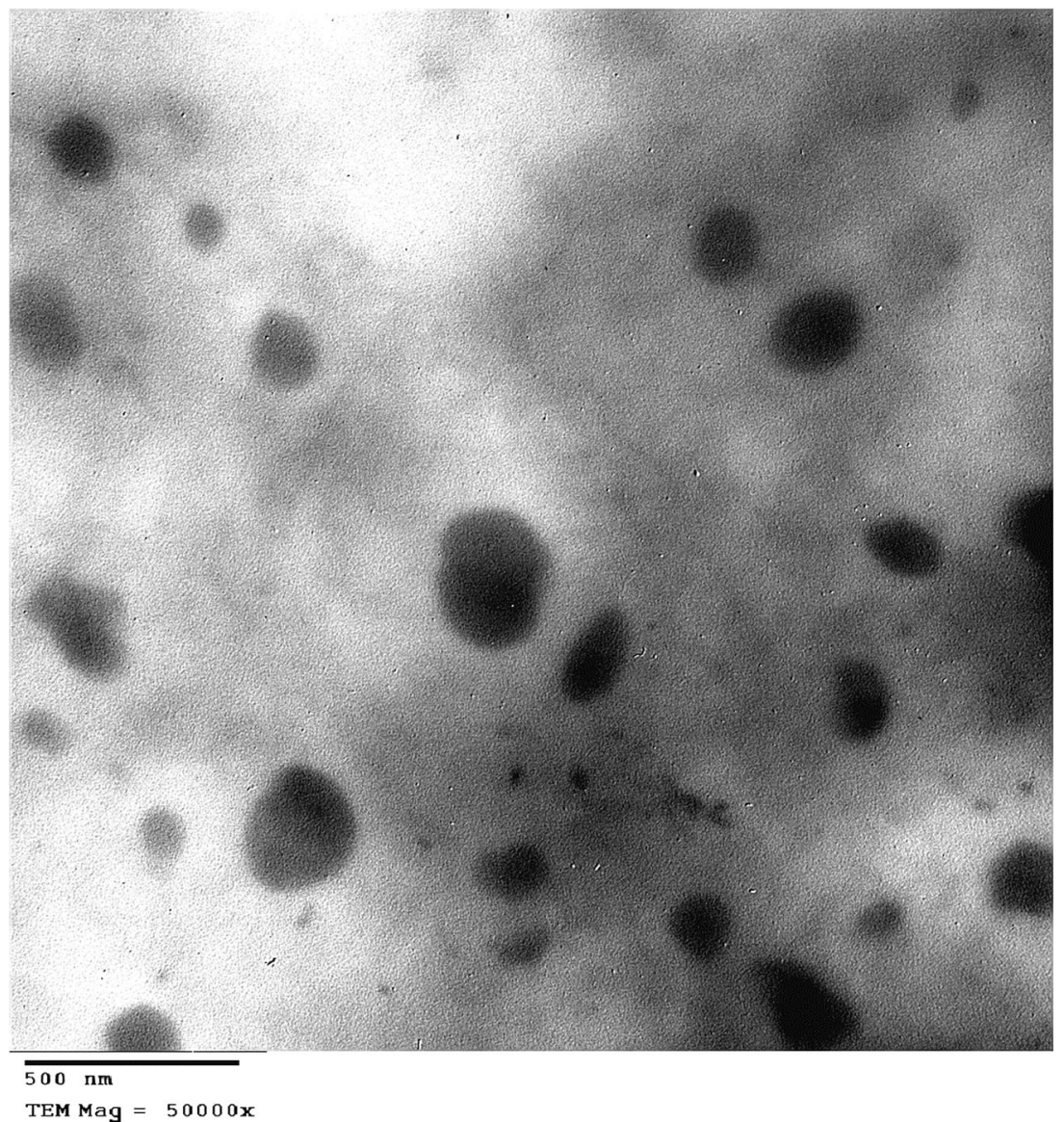

Fig. 2. Transmission electron image of TQ-loaded NLCs

\section{Pharmacokinetic Parameters of $T Q$}

The results from the bioavailability study are given in Fig. 3 and Table III. The experimental results revealed that both F9 and F12 showed elevated drug concentrations in plasma in comparison with suspension $(P<0.05)$. Moreover, the $C_{\max }$ values of TQ in rats treated with F9 and F12 were $3237 \pm 569.91$ and $3342 \pm 224.76 \mathrm{ng} / \mathrm{ml}$, respectively, with $T_{\max }$ of

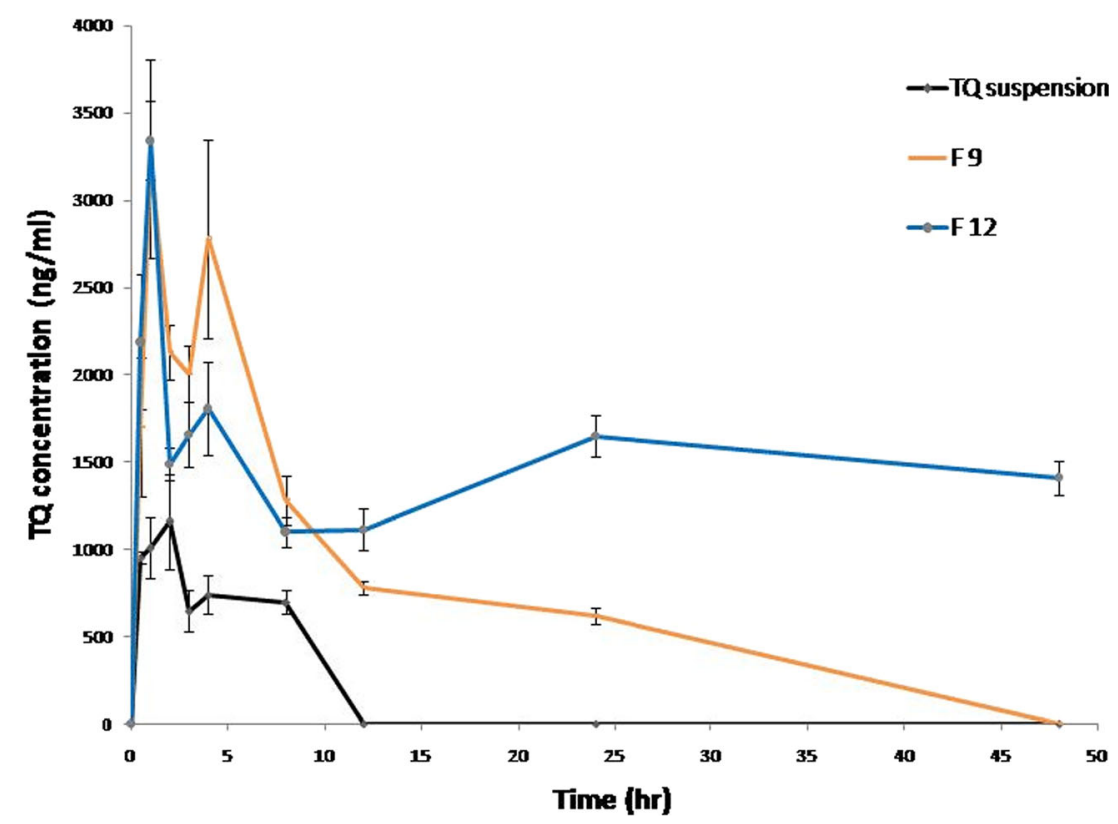

Fig. 3. Plasma concentration $v s$. time plotting after oral administration of TQ suspension, F9, and F12. Each value represents the mean \pm SD $(n=5)$ 
Table III. Pharmacokinetic Parameters of TQ Suspension, F9 and F12 (mean \pm SD, $n=5)$

\begin{tabular}{llll}
\hline Parameters & TQ suspension & F9 & F12 \\
\hline$C_{\max }(\mathrm{ng} / \mathrm{ml})$ & $1160.5 \pm 270.85$ & $3237 \pm 569.91$ & $3342 \pm 224.76$ \\
$T_{\max }(\mathrm{h})$ & 2 & 1 & 1 \\
$\mathrm{AUC} C_{0-t}(\mu \mathrm{g} \mathrm{h} / \mathrm{ml})$ & $17.919 \pm 2.4$ & $36.51 \pm 6.15$ & $71.19 \pm 7.94$ \\
$\mathrm{Ke}\left(\mathrm{h}^{-1}\right)$ & $0.151 \pm 0.045$ & $0.04 \pm 0.005$ & $0.0067 \pm 0.0002$ \\
$t_{1 / 2}(\mathrm{~h})$ & $4.6 \pm 1.14$ & $17.3 \pm 3.65$ & $102.7 \pm 16.74$ \\
$\mathrm{Fr}$ & - & 2.03 & 3.97 \\
\hline
\end{tabular}

approximately $1 \mathrm{~h}$. These results showed faster absorption $(1 \mathrm{~h}$ earlier) and significant higher plasma concentrations $(P<0.05)$ than TQ suspension. The clear difference between $T_{\max }$ values of TQ-loaded NLCs and TQ suspension confirmed that the rates of absorption of two systems were different. Faster absorption of F9 and F12 was because incorporation of TQ into lipid-based nanoparticles helped in avoiding passing extensive gut wall metabolism due to intimate association of drug with lipid and absorption through oral lymphatic region (26). As the pharmacological effects of TQ directly depended upon the plasma concentration, F9 and F12 could be more effective than TQ suspension in clinical experiments. Twelve hours after TQ suspension (oral administration), TQ was not detected in plasma. There was a clear difference in the $\mathrm{AUC}_{0-t}$ between suspension $(17.919 \pm 2.4 \mu \mathrm{g} \mathrm{h} / \mathrm{ml})$ and NLC formulations $(36.51 \pm 6.15$ and $71.19 \pm 7.94 \mu \mathrm{g} \mathrm{h} / \mathrm{ml}$ for F9 and F12, respectively). The $\mathrm{AUC}_{0-t}$ values for the drug were higher in the animals administered with NLCs, with a relative bioavailability of 2.03- and 3.97-fold (for F9 and F12, respectively) higher than TQ suspension, indicating the improved bioavailability of the drug in the lipid-based nanoparticles formulation. Additionally, half lives of NLC formulations were significantly $(P<0.05)$ increased while elimination rate constants were significantly $(P<0.05)$ decreased when compared with suspension. These results collectively indicated significant improvement of systemic absorption of TQ when loaded into NLC when compared with TQ suspension.

As poor aqueous solubility, excessive hepatic first pass effect and the separation of formulations from the aqueous media of intestinal contents are major obstacles to absorption of poorly water-soluble lipid products. NLCs are considered as a potential carrier for enhancing oral bioavailability of poorly water-soluble drugs. In general, different mechanisms have been documented for the absorption of the NLC from the intestine. One of these mechanisms is direct uptake through the GI tract which is attributed to small particle size and lipid content. The particle size of the NLC formulations was almost less than $200 \mathrm{~nm}$, and this reduced particle size increased affects the surface area of the NLCs. This small size permits better uptake in the intestine particularly in the lymphatic region of the tissue thus avoiding hepatic first pass metabolism (27). NLC was composed of solid and liquid lipids which were structurally similar to fat rich in food. The lipids could induce bile secretion in the small intestinal, and NLCs were associated with bile salt to form mixed micelles which helped the intact NLCs get into the lymphatic vessels and avoid the liver first pass metabolism (28). The uptake and lymphatic transport of intact NLCs are major factors in the promoted absorption. Another mechanism that supports absorption of NLCs is an increase in permeability by surfactants. Surfactants might increase the intestinal epithelial permeability by disturbing the cell membrane and reversibly open the tight junction of intestinal epithelial cell (29) and hence facilitate paracellular absorption. Concerning our formulations, F9 contains Pluronic F127 as surfactant which affects intestinal epithelial permeability and prolongs intestinal residence time. Pluronic is documented to deform the cell membrane and open the tight junction of the intestinal epithelial cell facilitating paracellular transport of NLCs (30). F12 contains Tween 80 as a surfactant, which is well documented as an absorption enhancer (31).

TQ exhibited double peak phenomenon in all curves and is not limited to NLC formulations. To our knowledge, no authors described TQ double-peak behavior except Beck et al. who showed that for $\mathrm{C}^{14}$-carvacrol (TQ is carvacrol drivative). This study supported our results as early first peak $(0.5 \mathrm{~h})$, indicating fast absorption, and was followed by a rapid decline. A second increase in the 2-8-h time period was most probably caused by re-uptake of carvacrol through enterohepatic recirculation (32).

Taking in our consideration F9 and F12, nearly similar first plasma peaks $(3237 \pm 569.9$ and $3342 \pm 224.7 \mathrm{ng} / \mathrm{ml}$, respectively) occurred at $1 \mathrm{~h}$ while second peak appeared at $4 \mathrm{~h}$ with higher plasma concentration of F9 than F12 (2780 \pm 571.7 and $1806 \pm 265.2 \mathrm{ng} / \mathrm{ml}$, respectively). Unexpectedly, F12 appeared in plasma at $48 \mathrm{~h}$ time point while F9 disappeared. This behavior of two NLC formulations might be attributed to their different compositions. As NLC formulations would be absorbed in an intact form through lymphatic vessels (28), F9 and F12 exhibited nearly similar early peaks. In case of F9, which contained Pluronic F127 (thermoreversible polymer), the second peak is the summation of early absorbed dose which undergone enterohepatic recycling and the delayed released dose from Pluronic containing system. Time later (at $48 \mathrm{~h}$ ), there was no detectable TQ in plasma as all dose has been released and completely excreted. Switching to F12 had been subjected to several times of enterohepatic recycling evidenced by higher plasma concentration at $24 \mathrm{~h}$ time point than $12 \mathrm{~h}$. Recurrent enterohepatic recycling prolonged the duration of F12 and TQ appeared at $48 \mathrm{~h}$.

As these findings confirmed the superiority of NLC formulations on suspension, pharmacological effects would be performed on F9 and F12 besides controls.

\section{Pharmacological Effects}

\section{Biochemical Changes}

In vivo hepatocurative affect of TQ NLCs was studied against paracetamol induced hepatotoxicity in Wister rats. The biochemical parameters (ALT, AST, and total bilirubin) of various experimental animal groups are given in Table IV. 
Table IV. Serum and Tissue Biochemical Parameters (mean $\pm \mathrm{SD}, n=5)$

\begin{tabular}{|c|c|c|c|c|c|c|}
\hline & ALT (units/ml) & AST (units/ml) & Albumin (g/dl) & Total bilirubin $(\mathrm{mg} / \mathrm{dl})$ & $\operatorname{MDA}(\mathrm{nM} / \mathrm{mg})$ & $\mathrm{GSH}(\mu \mathrm{M} / \mathrm{g})$ \\
\hline Group I & $190.7 \pm 15.55$ & $253.3 \pm 22.65$ & $2.445 \pm 0.062$ & $2.940 \pm 0.061$ & $500.9 \pm 4.55$ & $8.332 \pm 0.01872$ \\
\hline Group II & $530.0 \pm 45.22$ & $472.3 \pm 24.55$ & $2.053 \pm 2.432$ & $3.220 \pm 0.035$ & $642.2 \pm 11.9$ & $7.845 \pm 0.025$ \\
\hline Group III & $277.3 \pm 20.14$ & $340.0 \pm 17.69$ & $2.432 \pm 0.041$ & $3.827 \pm 0.035$ & $503.6 \pm 22.22$ & $8.308 \pm 0.004$ \\
\hline Group IV & $305.0 \pm 24.88$ & $304.7 \pm 23.55$ & $2.560 \pm 0.089$ & $2.893 \pm 0.074$ & $492.7 \pm 16.15$ & $8.369 \pm 0.071$ \\
\hline
\end{tabular}

Paracetamol in large dose is recognized to produce severe liver damage which can be identified by a significant increase in the marker enzymes ALT and AST level $(P<0.05)$ compared with that of the control group. As ALT and AST are normally present in high concentration in hepatocytes, their presence in high concentrations in circulation indicates damage of hepatocytes or their membranes (33). Elevation of total bilirubin reflected the depth of jaundice while the lowered level of albumin was attributed to damage of endoplasmic reticulum and loss of $\mathrm{P} 450$ leading to its functional failure and decrease in protein synthesis (34).

Animals treated with F9 (group III) showed a significant $(P<0.05)$ decrease in serum ALT to $277.3 \pm 20.14 \mathrm{U} / \mathrm{ml}$ when compared with untreated toxic group (group II) while showing significant $(P<0.05)$ elevation of albumin $(2.432 \pm 0.041 \mathrm{~g} / \mathrm{dl})$ and reached nearly to normal value $(2.445 \pm 0.062 \mathrm{~g} / \mathrm{dl})$. As
ALT catalyses, the conversion of alanine to pyruvate and glutamate is more specific to the liver and is thus a better parameter for detecting liver injury. The rise in albumin level indicated stabilization of endoplasmic reticulum leading to protein synthesis. Additionally, insignificant reduction in AST and bilirubin was observed in results of animals treated with F9 when compared with untreated toxic group.

Animals treated with F12 (group IV) showed significant $(P<0.05)$ decrease in both serum ALT and AST to reach 305.0 \pm 24.88 and $304.7 \pm 23.55 \mathrm{U} / \mathrm{ml}$, respectively, when compared with untreated toxic group (group II). This restoration of these markers could be due to stabilization of the membranes thereby preventing the leakage of intracellular enzymes. This is in agreement with the commonly accepted view that serum levels of transaminases return to normal with the healing of hepatic parenchyma and the regeneration of hepatocytes (35).
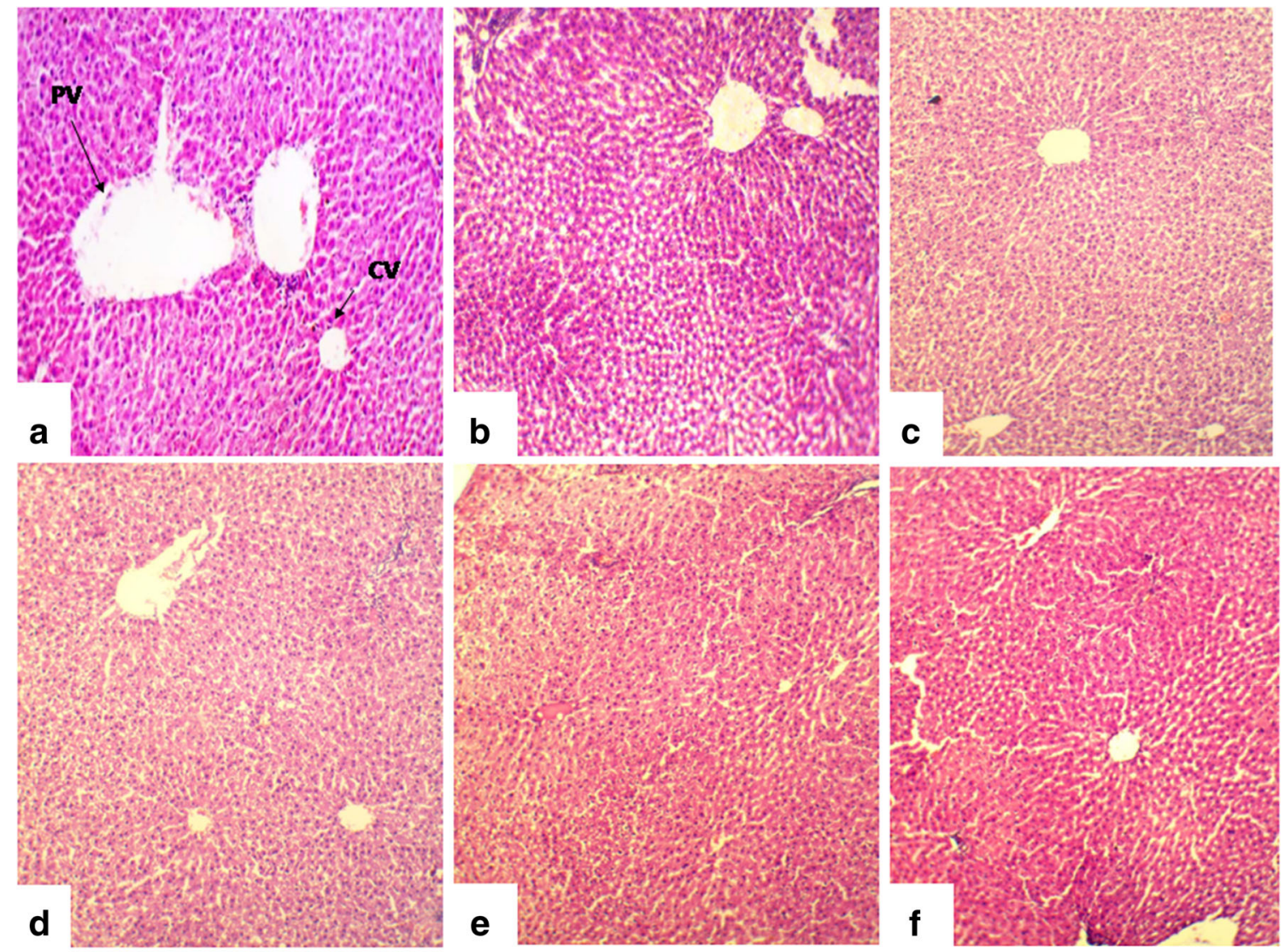

Fig. 4. a Histopathology of group I treated with normal saline $(1 \mathrm{ml} / \mathrm{kg})$ only. b Histopathology of group II treated with paracetamol $(800 \mathrm{mg} / \mathrm{kg})$. c, d Histopathology of group III treated with F9 and paracetamol $(800 \mathrm{mg} / \mathrm{kg})$. e, f Histopathology of group IV treated with F12 and paracetamol $(800 \mathrm{mg} / \mathrm{kg})$ 
By comparing with group I, values of albumin $(2.560 \pm 0.089 \mathrm{~g} /$ dl) and total bilirubin $(2.893 \pm 0.074 \mathrm{mg} / \mathrm{dl})$ insignificantly changed which indicated stabilization of endoplasmic reticulum and biliary dysfunction induced by paracetamol toxicity. These results indicated superiority of F12 in reducing hepatic tissue damage according to biochemical changes.

\section{Tissue Biochemical Assay}

MDA, the end products of lipid peroxidation in the liver tissue, is important indicators of tissue anti-oxidant defense mechanisms. High levels of MDA indicate tissue damage and failure of anti-oxidant defense mechanisms to prevent the formation of excessive free radicals (36). GSH, the major non-protein thiol in living organisms removes free radical species such as hydrogen peroxide, superoxide radicals and maintains membrane protein thiols depleted in hepatic mitochondria during hepatic injury due to toxins. As shown in Table IV, group II exhibited significant $(P<0.05)$ higher level of MDA (measured by TBARS levels) when comparing with group I which indicated lipid peroxidation and degradative process of membranous lipids, in liver tissue treated with paracetamol. Group II also showed significant $(P<0.05)$ lower level of GSH when comparing with group I which indicated marked oxidative injury due to its conjugation with NAPQI ( $N$-acetyl parabenzoquinoneimine paracetamol metabolite) to form mercapturic acid (37).

Administration of F9 (group III $503.6 \pm 22.22 \mathrm{nM} / \mathrm{mg}$ ) and F12 (group IV $492.7 \pm 16.15 \mathrm{nM} / \mathrm{mg}$ ) showed significant $(P<0.05)$ decline of MDA levels when comparing with group II which indicated marked restoration of lipid peroxidation towards their normal values. They also showed significant $(P<0.05)$ elevation of GSH levels (F9 $8.308 \pm 0.004$ and F12 $8.369 \pm 0.071 \mu \mathrm{M} / \mathrm{g}$ ) when comparing with group II which indicated their ability to reduce oxidative stress.

Our studies showed that the treatment of animals with F9 and F12 significantly restored the metabolic enzyme activities which indicate they improved the physiological functions in liver tissue. Accordingly, further histopathological studies were performed to investigate the efficacy on improving liver histology.

\section{Histopathological Examination}

Hepatic fibrosis is usually initiated by hepatocyte damage. Biological factors such as hepatitis virus, bile duct obstruction, cholesterol overload, etc. or chemical factors (such as administration of paracetamol in toxic dose), alcohol intake are known to contribute to liver fibrosis (38).

Figure $4 \mathrm{a}-\mathrm{f}$ depicted the photomicrograph of histopathological section of liver tissue from different groups. Histopathological examinations provided supportive results for the biochemical analysis. Group I (Fig. 4a) showed regular liver architecture without any apparent histopathological alteration in the structure of the central vein $(\mathrm{CV})$, portal vein $(\mathrm{CV})$ and surrounding hepatocytes in the cords with normal sinusoidal spaces and portal tract. In contrast, photomicrographs of histopathological liver sections of group II (Fig. 4b; intoxicated with paracetamol large dose) showed abnormal hepatic cells represented by massive fatty change liver and severe portal inflammation. Multinecrotic cells, piknotic heaptocytes, rupture and congestion in central vein with marked hemorrhage and appeared of melanomacrophage cells were recorded.

Hepatic sections of group III animals (Fig. 4c, d; animals treated with F9) revealed recovery of liver tissues represented by more normal architecture of the liver tissue with minimal inflammation and hemolysis in hepatic cells, but the most histological structures of liver still normal like liver in the control group.

Hepatic sections of group IV animals (Fig. 4e, f; animals treated with F12) showed moderate improvement in the histological structures of liver and became like that in liver tissues of the control group. Moderate histopathological effects included mild congestion in central vein with normal structure of hepatic cells and hepatic polygonal cells appeared with round nucleus.

\section{CONCLUSION}

We have successfully prepared and characterized TQloaded NLCs for enhancement of oral delivery and bioavailability of TQ. The encapsulation efficiency, particle size, PDI and physical stability of TQ into the NLCs were affected by the system used. Gelucire 43/01 Tween 80 seemed to be more stable than other investigated systems specially Compritol 888 ATO Pluronic F127 system which showed the poorest physical homogeneity. Pharmacokinetic study showed that $\mathrm{AUC}_{0-t}$ values for the drug, and consequently, relative bioavailability was higher in the animals administered with NLCs than TQ suspension. Besides, half lives of NLC formulations were significantly $(P<0.05)$ increased while elimination rate constants were significantly $(P<0.05)$ decreased when compared with suspension indicating marked enhancement of TQ bioavailability. Pharmacological effects (including serum and tissue biochemical levels) of chosen formulations; F9 and F12 showed significant improvement of most liver biomarkers and anti-oxidant power. Histopathological examination of liver sections of F9 and F12 depicted correction of most injuries caused by toxic dose of paracetamol. Liver tissues appeared to be relatively normal with restored normal structure without necrotic changes even though minor dilatation and congestion were observed in the portal vein area.

\section{REFERENCES}

1. Sayed MD. Traditional medicine in health care. J Ethnopharmacol. 1980;2:19-22.

2. Woo CC, Kumar AP, Sethi G, Tan KH. Thymoquinone: potential cure for inflammatory disorders and cancer. Biochem Pharm. 2012;83:443-51.

3. Mansour MA. Protective effects of thymoquinone and desferrioxamine against hepatotoxicity of carbon tetrachloride in mice. Life Sci. 2000;66:2583-91.

4. Al-Maje A, Al-Omar F, Nagi M. Neuroprotective effects of thymoquinone against transient forebrain ischemia in the rat hippocampus. Eur J Pharmacol. 2006;543:40-7.

5. El-Mahmoudy A, Shimizu Y, Shiina T, Matsuyama H, El-Sayed M, Takewaki T. Successful abrogation by thymoquinone against induction of diabetes mellitus with streptozotocin via nitric oxide inhibitory mechanism. Int Immunopharmacol. 2005;5:195-207.

6. El Gazzar M, El Mezayen R, Marecki JC, Nicolls MR, Canastar A, Dreskin SC. Antiinflammatory effect of thymoquinone in a mouse model of allergic lung inflammation. Int Immunopharmacol. 2006;6:1135-42.

7. Badary OA, Abd-Ellah MF, El-Mahdy MA, Salama SA, Hamada FM. Anticlastogenic activity of thymoquinone against benzo(a)pyrene in mice. Food Chem Toxic. 2007;45:88-92. 
8. Gali MH, Roessner A, Schneider-Stock R. Thymoquinone: a promising anticancer drug from natural sources. Int J Biochem Cell Biol. 2006;38:1249-53.

9. Oguz S, Kanter M, Erboga M, Erenoglu C. Protective effects of thymoquinone against cholestatic oxidative stress and hepatic damage after biliary obstruction in rats. J Mol Histol. 2012;43:151-9.

10. O'Driscoll CM, Griffin BT. Biopharmaceutical challenges associated with drugs with low aqueous solubility - the potential impact of lipid-based formulations. Adv Drug Deliv Rev. 2008;60:617-24.

11. Saupe A, Wissing SA, Lenk A, Schmidt C, Müller RH. Solid lipid nanoparticles (SLN) and nanostructured lipid carriers (NLC) structural investigations on two different carrier systems. Biomed Mater Eng. 2005;15:393-402.

12. Müller RH, Petersen RD, Hommoss A, Pardeike J. Nanostructured lipid carriers (NLC) in cosmetic dermal products. Adv Drug Deliv Rev. 2007;59:522-30.

13. Charman WN. Lipids, lipophilic drugs and oral drug delivery some emerging concepts. J Pharm Sci. 2000;89:967.

14. Porter $\mathrm{CJH}$, Charman WN. Intestinal lymphatic drug transport an update. Adv Drug Deliv Rev. 2001;50:61.

15. Najmi AK, Pillai KK, Pal SN, Aqil M. Free radical scavenging and hepatoprotective activity of jigrine against galactosamine induced hepatopathy in rats. J Ethnopharmacol. 2005;97:521-5.

16. Mihara M, Uchiyama M. Determination of malonaldehyde precursor in tissues by thiobarbituric acid test. Anal Biochem. 1978;86:271-8.

17. Beutler E, Duron O, Kelly BM. Improved method for the determination of blood glutathione. J Lab Clin Med. 1963;61:882-8.

18. El-Samaligy MS, Afifi NN, Mahmoud EA. Evaluation of hybrid liposomes-encapsulated silymarin regarding physical stability and in vivo performance. Int J Pharm. 2006;319:121-9.

19. Souto EB, Wissing SA, Barbosa CM, Müller RH. Development of a controlled release formulation based on SLN and NLC for topical clotrimazole delivery. Int J Pharm. 2004;278:71-7.

20. Puglia C, Blasi P, Rizza L, Schoubben A, Bonina F, Rossi C, et al. Lipid nanoparticles for prolonged topical delivery: an in vitro and in vivo investigation. Int J Pharm. 2008;357:295-304.

21. Hu LD, Xing T, De Cui F. Solid lipid nanoparticles to improve oral bioavailability of poorly soluble drugs. J Pharm Pharmacol. 2004;56:15-27.

22. Li Y, Taulier N, Rauth AM, Wu XY. American Association of Pharmaceutical Scientists (AAPS) conference, TN, USA, Nov. 2005; 7-11.

23. Siekmann B, Westesen K. Submicron-sized parenteral carrier systems based on solid lipids. Pharm Pharmacol Lett. 1992;1:123-6.
24. Ahlin P, Kristl J, Korbors JS. Optimization of procedure parameters and physical stability of solid lipid nanoparticles in dispersions. Acta Pharm. 1998;48:259.

25. Zhang L, Han L, Sun X, Gao D, Qin J, Wang J. The use of PEGylated liposomes to prolong the circulation lifetime of salvianolic acid B. Fitoterapia. 2012;83:678-89.

26. Ravi PR, Aditya N, Kathuria H, Malekar, Vats SR. Lipid nanoparticles for oral delivery of raloxifene: optimization, stability, in vivo evaluation and uptake mechanism. Eur J Pharm Biopharm. 2014;87:114-24.

27. Tiwari R, Pathak K. Nanostructured lipid carrier versus solid lipid nanoparticles of simvastatin: comparative analysis of characteristics, pharmacokinetics and tissue uptake. Int J Pharm. 2011;415:232-43.

28. Jacobs C, Kayser O, Müller RH. Nanosuspensions as a new approach for the formulation for the poorly soluble drug tarazepide. Int J Pharm. 2000;196:161-4.

29. Lindmark T, Nikkila T, Artursson P. Mechanisms of absorption enhancement by medium chain fatty acids in intestinal epithelial Caco-2 cell monolayers. J Pharmacol Exp Ther. 1995;275:958-64.

30. Wu G, Lee KYC. Effects of Poloxamer 188 on phospholipid monolayer morphology: an atomic force microscopy study. Langmuir. 2009;25:2133-9.

31. Aungst BJ. Absorption enhancers: applications and advances. AAPS. 2012;14:10-8.

32. Eisenbrand G, Hengstler J, Joost H-G, Kulling S, Rietjens I, Schlatter J, et al. In: Beck M, Bruchlen M, Elste V, Mair P, Rümbeli R, editors. Risk assessment of phytochemicals in food novel approaches. Weinheim: Wiley-VCH Verlag $\mathrm{GmbH} \& \mathrm{Co}$. $\mathrm{KGaA}$; 2010. p. 369-70.

33. Kew MC. Serum aminotransferase concentration as evidence of hepatocellular damage. Lancet. 2000;355:591-2.

34. Dolai N, Karmakar I, Kumar RBS, Kar B, Bala A, Haldar PK. Free radical scavenging activity of Castanopsis indica in mediating hepatoprotective activity of carbon tetrachloride intoxicated rats. Asian Pac J Trop Biomed. 2012; S243-51.

35. Vadivu R, Krithika A, Biplab C, Dedeepya P, Shoeb N, Lakshmi KS. Evaluation of hepatoprotective activity of the fruits of Coccinia grandis Linn. Int J Health Res. 2008;1:163-8.

36. Halliwell B, Gutteridge JMC. Free radicals in biology and medicine. 2nd ed. Oxford: Clarendon Press; 1989. p. 416.

37. Ozer J, Ratner M, Shaw M, Bailey W, Schomaker S. The current state of serum biomarkers of hepatotoxicity. J Toxicol. 2008;245:194-205.

38. Baravalia Y, Vaghasiya Y, Chanda S. Hepatoprotective effect of Woodfordia fruticosa Kurz flowers on diclofenac sodium induced liver toxicity in rats. Asian Pac J Trop Med. 2011;4:342-6. 